\title{
Biasing Selection Contests with Ex-Ante Identical Agents*
}

\author{
Kohei Kawamura ${ }^{\dagger} \quad$ Inés Moreno de Barreda ${ }^{\ddagger}$ \\ February 2014
}

\begin{abstract}
This note shows that when the designer of a contest wishes the winner to have high ability, she is better off giving a head start to one of the contestants even if they are ex-ante identical. If the contestants are ex-ante asymmetric, the designer should give a head start to the one who is more likely to have high ability.
\end{abstract}

Keywords: Contest, Selection, Head start, Favouritism

JEL Codes: C72, D82

\section{Introduction}

In many cases of interest, the winner of a contest is expected to have higher ability than other contestants. Examples include architectural design competitions, competitive research grants, and recruitment and promotion of staff. In this note we demonstrate that when the objective of the designer of an all-pay contest is to select a contestant with high ability, the designer is better off giving an advantage to one of the contestants even if they are ex-ante identical from her viewpoint. We also show as a corollary that if the contestants are ex-ante asymmetric, the designer should give a head start to the one who is more likely to have high ability.

Selection in contests was first studied by Mever (1991) who obtained related results to ours in a dynamic statistical (rather than game theoretic) model with two non-strategic agents. Unlike Mever (1991), this note considers selection contests in a static one-shot game with fully strategic agents 1 The economic insight from our approach is distinct and novel. 2 In particular, since our model is simple but features strategic agents, our insight could easily be contrasted to or incorporated into other models of all-pay contests 3

\section{Model}

Consider an all-pay contest with two agents, who have either high ability or low ability. While the agents know each other's ability, the principal cannot directly observe it. For expositional

\footnotetext{
${ }^{*}$ We thank Margaret Meyer, Benny Moldovanu, Santiago Sánchez-Páges, Cédric Wasser, an anonymous referee, and seminar participants at Bonn, Edinburgh and Kyoto for helpful comments and discussions. All errors are our own.

${ }^{\dagger}$ School of Economics, University of Edinburgh. kohei.kawamura@ed.ac.uk

${ }^{\ddagger}$ Nuffield College, University of Oxford. ines.morenodebarreda@economics.ox.ac.uk

${ }^{1}$ In Mever 1991) it is never optimal to bias a one-shot contest when the agents are ex-ante identical.

${ }^{2}$ Other contributions to the literature on selection contests with strategic agents include Clark and Riis (2001) and Münster (2007) but they consider different design problems from ours and do not obtain unequal treatment of ex-ante identical agents.

[ Konrad 2009) offers an extensive overview on the effort maximizing contest literature. Recently Kirkegaard 2012) and Pérez-Castrillo and Wettstein 2013) have shown that unequal treatment of (ex-ante and ex-post) symmetric contestants may be beneficial in settings where the designer is concerned with the contestants' effort.
} 
convenience we assume that one of the agents has high ability and the other has low ability. Our model can easily be modified to incorporate independent type distributions without changing the main insight.

Before the contest takes place, one of the agents is given a head start $a>0$, and then each agent exerts effort $e_{i} \geq 0, i \in\{L, H\}$. In the contest, the one who generates the highest "score" is declared the winner and obtains a fixed reward $V$, while the reward for the other agent is normalized to 0.4 The score of an agent is the sum of his effort and head start $a>0$, if he has any. That is, the score of agent $i$ is given by

$$
s_{i}= \begin{cases}e_{i}+a & \text { if agent } i \text { has head start } \\ e_{i} & \text { if agent }-i \text { has head start. }\end{cases}
$$

Each agent's winning probability $p\left(s_{i}, s_{-i}\right), i \in\{H, L\}$, is given by

$$
p_{i}\left(s_{i}, s_{-i}\right)= \begin{cases}1 & \text { if } s_{i}>s_{-i} \\ 0 & \text { if } s_{i}<s_{-i} \\ p_{i} \in[0,1] & \text { if } s_{i}=s_{-i}\end{cases}
$$

We assume that the effort cost is lower for the high ability agent. In particular, denoting $c_{H}(\cdot)$ the effort cost function of the agent with high ability and $c_{L}(\cdot)$ that of the agent with low ability, we assume that $c_{i}(0)=0, c_{i}^{\prime}(\cdot)>0, c_{i}^{\prime \prime}(\cdot) \geq 0$ and $c_{H}(\cdot) \leq c_{L}(\cdot)$ for any given effort level 5 We further assume $c_{H}^{\prime \prime \prime}(\cdot) \leq 0$, that is, the third derivative of the cost function of the high ability agent is non-positive, which covers e.g., linear and quadratic effort costs. We will discuss this assumption when interpreting our result later. The payoff function of the agent with high ability and that of the agent with low ability are given respectively by

$$
u^{H}=\left\{\begin{array}{ll}
V-c_{H}\left(e_{H}\right) & \text { if the agent wins } \\
-c_{H}\left(e_{H}\right) & \text { if the agent loses; }
\end{array} \text { and } u^{L}= \begin{cases}V-c_{L}\left(e_{L}\right) & \text { if the agent wins } \\
-c_{L}\left(e_{L}\right) & \text { if the agent loses. }\end{cases}\right.
$$

The objective of the principal/contest designer is to maximize the probability that the agent with high ability wins the contest. Note that the head start is given by the principal to a particular agent ex-ante. Since she is not informed of each agent's type, she cannot be certain whether she has given the head start to the low or high ability agent.

Throughout this note we will refer as an illustration to the case of linear costs, where

$$
c_{H}\left(e_{H}\right)=e_{H} \text { and } c\left(e_{L}\right)=c e_{L} \text { with } c>1 \text {. }
$$

Definition. The selection power of a contest is the probability that the high ability agent achieves a higher score in equilibrium.

\footnotetext{
${ }^{4}$ In this note we take $V$ and $a$ as exogenous variables, in order to highlight whether to use a head start, and if so, which agent should receive it. In reality the level of the reward and that of the head start may indeed be difficult for the contest designer to control. The reward may involve non-monetary components such as honour and prestige that cannot be fine-tuned. The judge of a contest who exercises favouritism (head start) for a contestant may not be the same person as the designer, in which case it may be impossible to control the size of the head start precisely.

${ }^{5}$ Instead of convex effort costs we can equivalently assume a concave score function with linear effort costs.
} 


\section{Bias for Selection}

In this section we first derive the equilibrium effort without head start $(a=0)$ as a benchmark. We then compute the equilibrium effort when the agent with high ability receives the head start $a>0$, and also when the agent with low ability receives the head start. Lastly we study the selection power of the contest from the viewpoint of the principal who is uncertain about the ability of the agents, and prove our main result.

\subsection{Unbiased contest $(a=0)$}

In this case, an agent's score represents his effort. Following Siegel (2009), in equilibrium the effort density of the low ability agent and the high ability agent is given by

$$
\begin{aligned}
f_{L}\left(e_{L}\right) & =\frac{1}{V} c_{H}^{\prime}\left(e_{L}\right) \quad \text { for } e_{L} \in\left(0, c_{L}^{-1}(V)\right] \text { with mass } 1-\frac{1}{V} c_{H}\left(c_{L}^{-1}(V)\right) \text { at } e_{L}=0, \\
f_{H}\left(e_{H}\right) & =\frac{1}{V} c_{L}^{\prime}\left(e_{H}\right) \quad \text { for } e_{H} \in\left[0, c_{L}^{-1}(V)\right],
\end{aligned}
$$

respectively. The winning probability of the high ability agent in this neutral contest is given by

$$
\begin{aligned}
P_{N} & =\int_{0}^{c_{L}^{-1}(V)} f_{H}\left(e_{H}\right) F_{L}\left(e_{H}\right) d e_{H}=\int_{0}^{c_{L}^{-1}(V)} \frac{1}{V} c_{L}^{\prime}\left(e_{H}\right)\left[\frac{1}{V} c_{H}\left(e_{H}\right)+1-\frac{1}{V} c_{H}\left(c_{L}^{-1}(V)\right)\right] \\
& =1-\frac{1}{V} c_{H}\left(c_{L}^{-1}(V)\right)+\frac{1}{V^{2}} \int_{0}^{c_{L}^{-1}(V)} c_{L}^{\prime}\left(e_{H}\right) c_{H}\left(e_{H}\right) d e_{H} .
\end{aligned}
$$

It is easy to check that, under the linear costs in (1), the probability of winning is given by

$$
P_{N}=1-\frac{1}{2 c}
$$

\subsection{Bias for high ability agent}

Let us consider the case in which the high ability agent receives the head start, namely $s_{H}=a+e_{H}$. Note that the "score density" of each agent for the competing range is the same as above. In Siegel (2009)'s terms the high ability agent's "reach" is higher, and thus we know that the low ability agent obtains the expected payoff of 0 , which also means his highest effort is $c_{L}^{-1}(V)$. It follows that in equilibrium,

$$
\begin{aligned}
f_{L}\left(e_{L}\right) & =\frac{1}{V} c_{H}^{\prime}\left(e_{L}-a\right) \text { for } e_{L} \in\left(a, c_{L}^{-1}(V)\right] \text { with mass } 1-\frac{1}{V} c_{H}\left(c_{L}^{-1}(V)-a\right) \text { at } e_{L}=0 \\
f_{H}\left(e_{H}\right) & =\frac{1}{V} c_{L}^{\prime}\left(e_{H}+a\right) \text { for } e_{H} \in\left(0, c_{L}^{-1}(V)-a\right] \text { with mass } \frac{1}{V} c_{L}(a) \text { at } e_{H}=0 .
\end{aligned}
$$

The winning probability of the high ability agent is given by

$$
\begin{aligned}
P_{H}(a) & =\int_{0}^{c_{L}^{-1}(V)-a} f_{H}\left(e_{H}\right) F_{L}\left(e_{H}+a\right) d e_{H}+\frac{1}{V} c_{L}(a)\left(1-\frac{1}{V} c_{H}\left(c_{L}^{-1}(V)-a\right)\right) \\
& =\int_{0}^{c_{L}^{-1}(V)-a} \frac{1}{V} c_{L}^{\prime}\left(e_{H}\right)\left[\frac{1}{V} c_{H}\left(e_{H}\right)+1-\frac{1}{V} c_{H}\left(c_{L}^{-1}(V)\right)\right] d e_{H}+\frac{1}{V} c_{L}(a)\left(1-\frac{1}{V} c_{H}\left(c_{L}^{-1}(V)-a\right)\right. \\
& =1-\frac{1}{V} c_{H}\left(c_{L}^{-1}(V)-a\right)+\frac{1}{V^{2}} \int_{a}^{c_{L}^{-1}(V)} c_{L}^{\prime}\left(e_{H}\right) c_{H}\left(e_{H}-a\right) d e_{H} .
\end{aligned}
$$


It is easy to check that, under the linear costs as in (1), the probability of winning is given by

$$
P_{H}(a)=1-\frac{1}{2 c}+\frac{c a^{2}}{2 V^{2}}
$$

Given the assumptions on the cost functions, the winning probability for the high ability agent, $P_{H}(a)$, is non-decreasing in the head start $a$ :

$$
\begin{aligned}
\frac{\partial P_{H}(a)}{\partial a} & =\frac{1}{V^{2}}\left(\int_{a}^{c_{L}^{-1}(V)} c_{L}^{\prime}(e) c_{H}^{\prime}(e-a) d e+V c_{H}^{\prime}\left(c_{L}^{-1}(V)-a\right)\right) \\
& =\frac{1}{V^{2}} \int_{a}^{c_{L}^{-1}(V)} c_{L}^{\prime}(e)\left(c_{H}^{\prime}\left(c_{L}^{-1}(V)-a\right)-c_{H}^{\prime}(e-a)\right) d e+\frac{1}{V^{2}} \int_{0}^{a} c_{L}^{\prime}(e) c_{H}^{\prime}\left(c_{L}^{-1}(V)-a\right) d e \\
& \geq 0
\end{aligned}
$$

where the first equality follows from the fact that $V=\int_{0}^{c_{L}^{-1}(V)} c_{L}^{\prime}(e) d e$ and the inequality follows from the monotonicity and the convexity of the costs functions.

\subsection{Bias for low ability agent}

Suppose now that the low ability agent receives the head start. In this case, the high ability agent's reach, (the maximum score at which his valuation for winning is zero) is $c_{H}^{-1}(V)$, whereas that of the low ability worker is $a+c_{L}^{-1}(V)$. For expositional ease let us focus on the case where the head start is not too large, namely $a \leq c_{H}^{-1}(V)-c_{L}^{-1}(V)$, so that the high ability agent's reach is still higher than that of the low ability agent and the low ability agent's expected payoff is 0.6 It follows that in equilibrium

$$
\begin{aligned}
f_{L}\left(e_{L}\right) & =\frac{1}{V} c_{H}^{\prime}\left(e_{L}+a\right) \text { for } e_{L} \in\left(0, c_{L}^{-1}(V)\right] \text { with mass } 1-\frac{1}{V}\left(c_{H}\left(c_{L}^{-1}(V)+a\right)-c_{H}(a)\right) \text { at } e_{L}=0 \\
f_{H}\left(e_{H}\right) & =\frac{1}{V} c_{L}^{\prime}\left(e_{H}-a\right) \text { for for } e_{H} \in\left[a, c_{L}^{-1}(V)+a\right] .
\end{aligned}
$$

The probability of the high ability agent winning in this case is given by

$$
\begin{aligned}
P_{L}(a) & =\int_{a}^{c_{L}^{-1}(V)+a} f_{H}\left(e_{H}\right) F_{L}\left(e_{H}-a\right) d e_{H} \\
& =\int_{a}^{c_{L}^{-1}(V)+a} \frac{1}{V} c_{L}^{\prime}\left(e_{H}-a\right)\left[\frac{1}{V}\left(c_{H}\left(e_{H}\right)-c_{H}(a)\right)+1-\frac{1}{V}\left(c_{H}\left(c_{L}^{-1}(V)+a\right)-c_{H}(a)\right)\right] \\
& =1-\frac{1}{V} c_{H}\left(c_{L}^{-1}(V)+a\right)+\frac{1}{V^{2}} \int_{0}^{c_{L}^{-1}(V)} c_{L}^{\prime}\left(e_{H}\right) c_{H}\left(e_{H}+a\right) d e_{H} .
\end{aligned}
$$

Interestingly, under the linear costs [1, we have

$$
P_{L}(a)=1-\frac{1}{2 c},
$$

${ }^{6}$ If $a>c_{H}^{-1}(V)-c_{L}^{-1}(V)$, then the low ability agent's reach is higher than that of the high ability agent and hence the high ability agent's expected payoff is 0 . It is easy to see that in that case the probability of winning for the high ability agent is

$$
P_{L}(a)=-\frac{1}{V^{2}} \int_{0}^{c_{H}^{-1}(V)-a} c_{L}(e) c_{H}^{\prime}(e) d e+\frac{1}{V}\left(c_{L}\left(c_{H}^{-1}(V)-a\right)\right),
$$

which is, similarly to the case where $a \leq c_{H}^{-1}(V)-c_{L}^{-1}(V)$, decreasing in $a$. 
which coincides with the probability of the high ability agent winning in an unbiased contest. In this case, the high ability agent exerts higher effort to compensate completely for the disadvantage from the head start on his opponent.

It is easy to check that the probability of the high ability winning is non-increasing in $a$ :

$$
\begin{aligned}
\frac{\partial P_{L}(a)}{\partial a} & =\frac{1}{V^{2}}\left(\int_{0}^{c_{L}^{-1}(V)} c_{L}^{\prime}(e) c_{H}^{\prime}(e+a) d e-V c_{H}^{\prime}\left(c_{L}^{-1}(V)+a\right)\right) \\
& =\frac{1}{V^{2}} \int_{0}^{c_{L}^{-1}(V)} c_{L}^{\prime}(e)\left(c_{H}^{\prime}(e+a)-c_{H}^{\prime}\left(c_{L}^{-1}(V)+a\right)\right) d e \\
& \leq 0
\end{aligned}
$$

where the inequality follows from the assumptions on the cost functions.

\subsection{Biasing ex-ante symmetric contest}

Suppose both agents have equal probability of having high ability.

Proposition. The principal is strictly better off by giving a head start $a \in\left(0, c_{H}^{-1}(V)-c_{L}^{-1}(V)\right)$ to one of the agents randomly than by running the unbiased contest.

Proof. The expected payoff of the principal who gives a head start randomly is proportional to $P_{H}+P_{L}$. The effect of the random head start on the principal's payoff is positive if $\frac{\partial P_{H}(a)}{\partial a}+\frac{\partial P_{L}(a)}{\partial a}>$ 0 . This indeed holds since

$$
\begin{aligned}
V^{2}\left(\frac{\partial P_{H}(a)}{\partial a}+\frac{\partial P_{L}(a)}{\partial a}\right)= & \int_{a}^{c_{L}^{-1}(V)} c_{L}^{\prime}(e)\left(c_{H}^{\prime}\left(c_{L}^{-1}(V)-a\right)-c_{H}^{\prime}(e-a)\right) d e+\int_{0}^{a} c_{L}^{\prime}(e) c_{H}^{\prime}\left(c_{L}^{-1}(V)-a\right) d e \\
& +\int_{0}^{c_{L}^{-1}(V)} c_{L}^{\prime}(e)\left(c_{H}^{\prime}(e+a)-c_{H}^{\prime}\left(c_{L}^{-1}(V)+a\right)\right) d e \\
> & \int_{a}^{c_{L}^{-1}(V)} c_{L}^{\prime}(e)\left(c_{H}^{\prime}\left(c_{L}^{-1}(V)-a\right)-c_{H}^{\prime}(e-a)\right) d e+\int_{0}^{a} c_{L}^{\prime}(e)\left(c_{H}^{\prime}\left(c_{L}^{-1}(V)-a\right)-c_{H}^{\prime}(2 a)\right) d e \\
& +\int_{a}^{c_{L}^{-1}(V)} c_{L}^{\prime}(e)\left(c_{H}^{\prime}(e+a)-c_{H}^{\prime}\left(c_{L}^{-1}(V)+a\right)\right) d e+\int_{0}^{a} c_{L}^{\prime}(e)\left(c_{H}^{\prime}(0)-c_{H}^{\prime}\left(c_{L}^{-1}(V)+a\right)\right) d e \\
= & -\int_{a}^{c_{L}^{-1}(V)} c_{L}^{\prime}(e)\left[\left(c_{H}^{\prime}\left(c_{L}^{-1}(V)+a\right)-c_{H}^{\prime}\left(c_{L}^{-1}(V)-a\right)\right)-\left(c_{H}^{\prime}(e+a)-c_{H}^{\prime}(e-a)\right)\right] d e \\
& -\int_{0}^{a} c_{L}^{\prime}(e)\left[\left(c_{H}^{\prime}\left(c_{L}^{-1}(V)+a\right)-c_{H}^{\prime}\left(c_{L}^{-1}(V)-a\right)\right)-\left(c_{H}^{\prime}(2 a)-c_{H}^{\prime}(0)\right)\right] d e \geq 0
\end{aligned}
$$

where the first inequality follows from the monotonicity and convexity of the cost functions. To see that the last inequality holds, first notice that the two expressions in square brackets in the last two lines both take the form of $\left(c_{H}^{\prime}(x+a)-c_{H}^{\prime}(x-a)\right)-\left(c_{H}^{\prime}(y+a)-c_{H}^{\prime}(y-a)\right)$, with $x>y$ from $e<c_{L}^{-1}(V)$. These expressions are non-positive since $c_{H}^{\prime}$ exhibits weakly decreasing differences in its argument, as implied by the assumption $c_{H}^{\prime \prime \prime}(\cdot) \leq 0$. Therefore, the entire expression in the last two lines is non-negative.

The Proposition has a natural intuition 7 Consider the linear cost case with a relatively small head start. If the agent with high ability receives the head start, then the low ability agent becomes "discouraged" and bids down (his maximum effort becomes lower and he exerts zero effort more often), which leads to an increase in $P_{H}$, the probability of the high ability agent winning. Meanwhile, if the low ability agent receives the head start, insofar as the high ability agent's reach is higher, he "bids up" to make up for the disadvantage (thanks to his lower marginal cost) and keeps $P_{L}$, his probability of winning, unchanged. Thus overall the selection power of the contest increases.

\footnotetext{
${ }^{7}$ While we have focused on $a \leq c_{H}^{-1}(V)-c_{L}^{-1}(V)$, the Proposition will hold for higher $a$, the upper bound of which can be derived by using $P_{L}$ in Footnote 6 . We omit this case as it complicates the exposition without further economic insight.
} 
The feature that the disadvantaged high ability agent "bids up" to keep his winning probability completely unchanged (when his reach is higher) depends on the linearity of the effort cost. If it is convex, "bidding up" becomes more costly at an increasing rate and his winning probability will be lower than in the unbiased benchmark. However, the Proposition proves that as long as the convexity of the effort cost diminishes or stays the same, i.e., if its third derivative is non-positive, our main insight is valid.

\section{Discussion}

It is straightforward to see that if each agent has a different probability of having high ability, the principal should also use a head start, and in particular she should give it to the agent who is more likely to have high ability. This is because, as we have seen so far, the benefit of using a biased contest comes from the increase in the probability of the high ability agent winning when he receives the head start.

Corollary. If the contestants are ex-ante asymmetric, the principal gives a head start $a \in\left(0, c_{H}^{-1}(V)-\right.$ $\left.c_{L}^{-1}(V)\right)$ to the agent with the higher probability of having high ability.

We have assumed that the sole objective of the principal is to maximize the selection power, in order to highlight the effect of bias on selection in contests. The principal may also care about effort: it can be wasteful as in the influence activity literature (e.g. Milgrom, 1988), or beneficial as in the standard contest literature. The insight we have developed here could well be adapted for extensions of existing models of contests to study situations where both effort and selection are in the contest designer's objective function.

\section{References}

Clark, D. J., AND C. RiIS (2001): "Rank-Order Tournaments and Selection," Journal of Economics, 73, 167-191.

KIRKEGAARD, R. (2012): "Favoritism in Asymmetric Contests: Head Starts and Handicaps," Games and Economic Behavior, 76(1), 226-248.

KonRAD, K. A. (2009): Strategy and Dynamics in Contests. Oxford University Press.

Meyer, M. A. (1991): "Learning from Coarse Information: Biased Contests and Career Profiles," Review of Economic Studies, 58(1), 15-41.

Milgrom, P. R. (1988): "Employment Contracts, Influence Activities, and Efficient Organization Design," Journal of Political Economy, 96(1), 42-60.

Münster, J. (2007): "Selection Tournaments, Sabotage and Participation," Journal of Economics and Management Strategy, 16 (4), 943-970.

PÉrez-CAstrillo, D., And D. Wettstein (2013): “Innovation Contests," Working paper, Barcelona Graduate School of Economics.

Siegel, R. (2009): "All-Pay Contests," Econometrica, 77(1), 71-92. 